\title{
Hall effects on the steady structure of the rotational layer at the dayside magnetopause
}

\author{
Lars G. Westerberg and Hans O. Åkerstedt \\ Division of Fluid Mechanics, Luleå University of Technology, SE-971 87 Luleå, Sweden
}

(Received 20 June 2007; accepted 28 August 2007; published online 31 October 2007)

\begin{abstract}
The influence of the Hall term in the generalized Ohm's law on the large-scale plasma flow during conditions of ongoing magnetic reconnection at the dayside magnetopause (MP) is investigated. Of special interest is the plasma flow behavior during the transition of the MP transition layer as the Hall effect grows in proportion to the viscous-resistive effects. The governing equations are solved approximately by an ordinary perturbation expansion in orders of large Reynolds and Lundqvist numbers. It is shown that the flow pattern is strongly dependent on the magnitude of the Hall parameter; as it approaches zero, the viscous-resistive solution is obtained, while for an ordering of the same magnitude as the resistivity/viscosity, the Hall effect begins to affect the flow structure severely. For an increasing value on the Hall parameter, oscillations are brought into the system, an effect that is enhanced with the magnitude of the Hall parameter. Furthermore, it is shown that as the Hall effect begins to dominate, the transition layer thickens. (C) 2007 American Institute of Physics. [DOI: 10.1063/1.2786071]
\end{abstract}

\section{INTRODUCTION}

Magnetic reconnection is a central process for the conversion of magnetic energy to kinetic energy in space, astrophysical, and stellar physical applications. It is a process that dominates the transfer of mass and momentum from the solar wind into the terrestrial magnetosphere. In recent studies, Westerberg and $\AA_{\text {kerstedt }}{ }^{1-4}$ have made analytical viscousresistive models of the large-scale effects of reconnection on the solar wind plasma during the transition of the dayside magnetopause (MP) boundary. Since the solar wind plasma is mainly collisionless, other nonideal effects such as the Hall term in the generalized Ohm's law should affect the flow to a greater extent than just viscosity and resistivity.

In this study, we extend the viscous-resistive threedimensional (3D) model $^{2}$ to also include effects from the Hall term. Much of the accomplished work during the past decade covering the Hall physical effects in connection with the dynamics of magnetic reconnection combines analytical models with numerical simulations. In addition to these approaches, multi-spacecraft measurements have also been utilized. The main objective with the investigations of the inclusion of the Hall term on the reconnection process is primarily to clarify to what extent the onset mechanism and the reconnection structure are dependent on the Hall physics. Recently, Craig et al. ${ }^{5}$ combined an analytical framework with magnetohydrodynamic (MHD) simulations to investigate the impact of Hall current contributions on flow-driven planar magnetic merging solutions. From a dynamical analysis they found that the Hall current affects the planar merging if the dimensionless Hall parameter (or normalized ion skin depth) satisfies $c_{H}^{2}>\eta$, where $\eta$ is the inverse Lundqvist number for the plasma. This result is also supported by the numerical experiments, which show that the nature of the merging changes dramatically when the Hall coupling satisfies $c_{H}^{2}>\eta$. Furthermore, they showed that from the coupling provided by the Hall term, the reconnection rate may be reduced due to the emergence of multiple current layers that can enhance the global Ohmic dissipation. This work was newly followed by Senananyake and Craig, ${ }^{6}$ who enhance the conclusion that the Hall currents can influence reconnection rates, Ohmic dissipation rates, and the geometry of the reconnecting fields when $d_{i}^{2}>\eta$, where $d_{i}$ is the ion skin depth. Furthermore, Breslau and Jardin ${ }^{7}$ compared two lines of thought regarding the physical process of fast magnetic reconnection, one based on the Sweet-Parker (SP) model and one in which the Hall and electron pressure terms in Ohm's law are included. The SP model yields resistivity-dependent reconnection with a thick, moderate-aspect-ratio current sheet. For a sheet thickness less than or of the order of the ion inertial length (skin depth) scale, the Hall effect will predominate. This produces true fast magnetic reconnection with a microscopic current sheet of unit aspect ratio and a distorted magnetic field. Huba and Rudakov ${ }^{8}$ investigated the dependence of the Hall reconnection rate on the thickness of the initial current layer length scale. It was concluded that the reconnection rate is weakly dependent on the layer width, but not on the scale length on which the electron frozen-in condition breaks down, as long as it is less than the ion skin depth and the system size.

In addition to the analytical/computational approaches above, the influence of the Hall term has also been addressed by Vaivads et al., ${ }^{9}$ who used the full potential of the Cluster fleet to investigate the microscale physics in the diffusion region, and especially the Hall current effects. They show that the large electric fields at the separatrices are balanced by the Hall term, thus establishing the role of the Hall term in forming the structure and dynamics of the diffusion region.

A majority of the work presented above investigated the Hall current influence on fast reconnection regimes, which dominate in the solar corona. Furthermore, the primary objective is to analyze the main change in field topology and 
current sheet dynamics on length scales of the order of the ion-skin depth. In this paper, we extend the region of interest to well outside the diffusion region as we investigate the influence of finite gyroradius effects that enter Ohm's law through the Hall term (in addition to viscosity and resistivity as nonideal effects) on the solar wind plasma flow through the MP boundary during conditions of ongoing magnetic reconnection. The main objective is to put the focus on the global effects of ongoing magnetic reconnection. Previous studies have been done covering the large-scale 2D incompressible/compressible flow, a development of the 3D analysis, and a coupling of the $3 \mathrm{D}$ analysis with results from MHD simulations. $1,3,4,10$

To motivate the inclusion of the Hall terms, we recall that the resistive form of Ohm's law in laboratory space is

$$
\mathbf{j}=\sigma(\mathbf{E}+\mathbf{u} \times \mathbf{B}),
$$

where $\sigma$ is the conductivity. For the case in which the collisional mean free path is small compared to the characteristic length scale $L$, Eq. (1) is a good approximation. For reconnection problems, however, the transition layer thickness $\Delta$ is often of the same order as the Larmor radius $r_{L}$ in higher density plasmas. This means in turn that $r_{L} \geqslant \Delta$ even though $r_{L} \ll L$. For this case, the resistive Ohm's law is not a complete description without the finite Larmor radius terms in the generalized Ohm's law. ${ }^{11}$ The generalized Ohm's law is ${ }^{12}$

$$
\frac{m_{e}}{n e^{2}} \frac{\partial \mathbf{j}}{\partial t}=\mathbf{E}+\mathbf{u} \times \mathbf{B}-\frac{1}{n e}(\mathbf{j} \times \mathbf{B})+\frac{1}{2 n e} \nabla p-\eta \mathbf{j},
$$

where $\eta=1 / \sigma$ is the resistivity and $p$ is the plasma pressure. The $\mathbf{u} \times \mathbf{B}$ term is the convective electric field and $\eta \mathbf{j}$ is the field associated with Ohmic dissipation caused by electronion collisions. The third term on the right-hand side is the Hall effect. In the present paper, we consider only the Ohmic dissipation term together with the Hall term and the pressure

$$
\mathbf{E}+\mathbf{u} \times \mathbf{B}=\eta \mathbf{j}+\frac{1}{n e}(\mathbf{j} \times \mathbf{B})+\frac{1}{2 n e} \nabla p .
$$

The outline of the present paper is as follows. In Sec. II, we present in detail the inclusion of the Hall terms in the generalized Ohm's law and how they add to the equations in the viscous-resistive model. ${ }^{2}$ This results in the equations describing the plasma flow through the MP. By solving these equations in Secs. III and IV, we obtain the flow velocity and magnetic field behavior during the transition of the MP. In Sec. V, we proceed by investigating the flow behavior for different values of the Hall parameter. Section VI wraps it all up with a summary and conclusions.

\section{OHM'S LAW WITH THE HALL TERM}

Having a three-dimensional flow, we let

$$
\begin{aligned}
& \mathbf{u}=u_{x} \hat{\mathbf{x}}+u_{y} \hat{\mathbf{y}}+u_{z} \hat{\mathbf{z}}, \\
& \mathbf{B}=B_{x} \hat{\mathbf{x}}+B_{y} \hat{\mathbf{y}}+B_{z} \hat{\mathbf{z}},
\end{aligned}
$$

where $\hat{\mathbf{x}}, \hat{\mathbf{y}}$, and $\hat{\mathbf{z}}$ represent a local plane coordinate system close to the reconnection line, with $\hat{\mathbf{x}}$ pointing in the normal direction of the MP, $\hat{\mathbf{z}}$ pointing in the tangential direction,

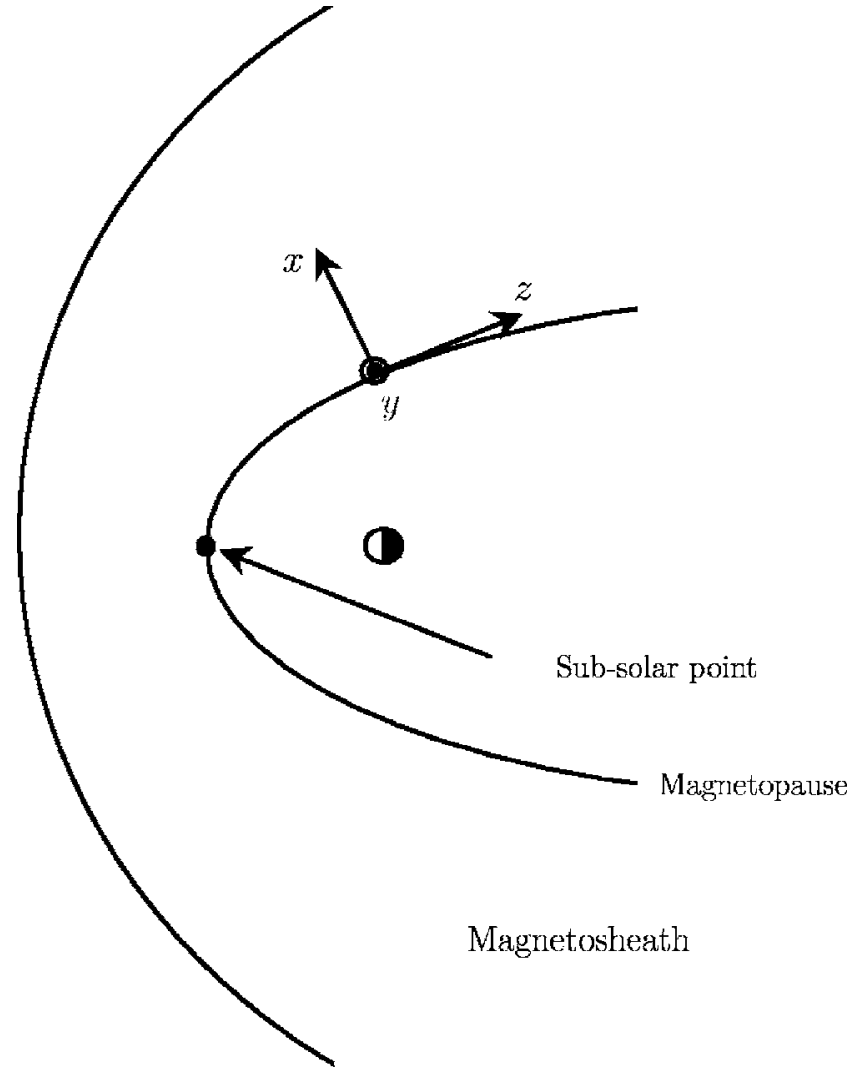

FIG. 1. The curvilinear coordinate system with conventional labels for the coordinate axis, with $x$ pointing in the normal direction of the MP surface, $z$ in the corresponding tangential direction, and $y$ completing the right-hand system. The coordinate system is hence identical to the local MP boundary coordinates (LMN) (Ref. 13), with $z=L, y=M$, and $x=N$.

and $\hat{\mathbf{y}}$ completing the right-hand system. These coordinates are identical to the corresponding local plane coordinates $L M N$ introduced by Russell and Elphic, ${ }^{13}$ such that $z=L$, $y=M$, and $x=N$. See Fig. 1 .

Considering Eq. (3), we write it on component form such that

$$
\begin{aligned}
& \hat{\mathbf{y}}: \quad E_{y}+u_{z} B_{x}-u_{x} B_{z}=\eta j_{y}+\frac{1}{n e}\left(j_{z} B_{x}-j_{x} B_{z}\right)-\frac{1}{2 n e} \frac{\partial p}{\partial y}, \\
& \hat{\mathbf{z}}: \quad E_{z}+u_{x} B_{y}-u_{y} B_{x}=\eta j_{z}-\frac{1}{n e} j_{y} B_{x}-\frac{1}{2 n e} \frac{\partial p}{\partial z}
\end{aligned}
$$

The MP transition layer separating magnetosheath plasma from plasma in the magnetosphere is assumed to be thin, so $\delta^{*}$ is introduced as a characteristic thickness. The corresponding characteristic length scale in the main flow directions $(\hat{\mathbf{y}}$ and $\hat{\mathbf{z}})$ is chosen as the Earth radius $\left(R_{E}\right)$. Introducing a small parameter $\delta$ such that

$$
\delta=\frac{\delta^{*}}{R_{E}},
$$

an order of magnitude estimate shows that as in ordinary boundary layer theory, the thickness scales as 


$$
\delta=O\left(R^{-1 / 2}\right) .
$$

Furthermore, it also follows that

$$
\frac{\partial}{\partial x}=O\left(R^{1 / 2}\right)
$$

Here $R$ is the Reynolds number

$$
R=\frac{v_{A} \cdot R_{E}}{\nu}
$$

where $v_{A}$ is the Alfvén velocity and $\nu$ is the kinematic viscosity. Dimensionless variables are introduced as follows:

$$
\begin{aligned}
& \frac{\mathbf{u}}{v_{A}} \rightarrow \mathbf{u}, \\
& \frac{\mathbf{B}}{B_{0}} \rightarrow \mathbf{B}, \\
& \frac{\mathbf{E}}{v_{A} B_{0}} \rightarrow \mathbf{E}, \\
& \frac{\mathbf{j}}{B_{0} \mu_{0} L} \rightarrow \mathbf{j}, \\
& \frac{\mathbf{r}}{L} \rightarrow \mathbf{r} .
\end{aligned}
$$

$\mathbf{r}=(x, y, z)$ is the position vector, $v_{A}$ is the Alfvén velocity at some reference point, for instance the reconnection site, $B_{0}$ is the corresponding magnetic field value, and $L$ is a characteristic length scale, which we choose in this analysis to be the Earth radius $R_{E}$. Considering Eq. (8), we introduce a new fast variable $\xi$ such that

$$
\begin{aligned}
& \xi=R^{1 / 2} x, \\
& \frac{\partial}{\partial x}=R^{1 / 2} \frac{\partial}{\partial \xi}, \\
& \frac{\partial^{2}}{\partial x^{2}}=R \frac{\partial^{2}}{\partial \xi^{2}} .
\end{aligned}
$$

Faraday's law

$$
\mu_{0} \mathbf{j}=\nabla \times \mathbf{B}
$$

gives

$$
\begin{aligned}
& \mu_{0} j_{\xi}=\frac{\partial B_{z}}{\partial y}-\frac{\partial B_{y}}{\partial z}, \\
& \mu_{0} j_{y}=\frac{\partial B_{\xi}}{\partial z}-R^{1 / 2} \frac{\partial B_{z}}{\partial \xi}, \\
& \mu_{0} j_{x}=R^{1 / 2} \frac{\partial B_{y}}{\partial \xi}-\frac{\partial B_{\xi}}{\partial y} .
\end{aligned}
$$

Combining Eqs. (5) and (13) and writing the variables in dimensionless form results in $\hat{\mathbf{y}}: \quad E_{y}+u_{z} B_{\xi}-u_{\xi} B_{z}=-\frac{R^{1 / 2}}{L_{u}} \frac{\partial B_{z}}{\partial \xi}+R^{1 / 2} c_{H} B_{\xi} \frac{\partial B_{y}}{\partial \xi}-\frac{c_{H}}{2} \frac{\partial p}{\partial y}$,

$\hat{\mathbf{z}}: \quad E_{z}+u_{\xi} B_{y}-u_{y} B_{\xi}=-\frac{R^{1 / 2}}{L_{u}} \frac{\partial B_{y}}{\partial \xi}+R^{1 / 2} c_{H} B_{\xi} \frac{\partial B_{z}}{\partial \xi}-\frac{c_{H}}{2} \frac{\partial p}{\partial z}$,

where $L_{u}$ and $c_{H}$ are the Lundqvist number and the Hall parameter, respectively, such that

$$
\begin{aligned}
L_{u} & =\frac{v_{A} R_{E}}{\eta_{d}}, \\
c_{H} & =\frac{B_{0}}{n e \mu_{0} v_{A} R_{E}},
\end{aligned}
$$

where $\eta_{d}$ is the magnetic diffusivity. Taking the curl of Eq. (14) and using Faraday's law together with the divergencefree property of the magnetic field results in the induction equation (24). Outside the transition layer, the magnetic and viscous diffusion terms are small. In the layer, however, the terms are balanced to be of the same order as the other terms. $R$ and $L_{u}$ are considered to be of the same order.

\section{The Hall parameter $c_{H}$}

In order to couple the Hall parameter $c_{H}$ in Eq. (15b) to the characteristic ion-skin depth (or ion-inertial length) $d_{i}$, we consider a general set of characteristic parameters $u_{0}, B_{0}$, and $L_{0}$, corresponding to the velocity, magnetic field, and length scale, respectively. $c_{H}$ can then be written as

$$
c_{H}=\frac{B_{0}}{n e \mu_{0} u_{0} L_{0}} .
$$

Using the Alfvén velocity $\left(v_{A}=B_{0} / \sqrt{\mu_{0} \bar{m}_{i} n}\right.$, where $\bar{m}$ is the mean ion particle mass) and the speed of light $\left(c=1 / \sqrt{\epsilon_{0} \mu_{0}}\right)$, we obtain

$$
c_{H}=\frac{v_{A}}{u_{0}} \cdot c \sqrt{\frac{\bar{m}_{i} \epsilon_{0}}{n e^{2}}},
$$

where the square root term is equal to the ion plasma frequency $\left(\omega_{p i}\right)$. Considering the characteristic velocity to be equal to the Alfvén velocity and the corresponding length scale to be the Earth radius, we obtain

$$
c_{H}=\frac{c}{\omega_{p i} R_{E}}=\frac{d_{i}}{R_{E}},
$$

where $d_{i}\left(=c / \omega_{p i}\right)$ is the ion-skin depth. Outside the transition layer, the Hall terms are therefore small and may be neglected. Inside the transition layer, the first of the two Hall terms may become large, and in order for this term to be important in the layer, $c_{H}$ is considered to be of order $O\left(R^{-1}\right)$.

\section{TRANSITION LAYER ANALYSIS}

In this section, we focus on how the inclusion of the Hall term contributes to the final equations governing the plasma flow through the MP under conditions of magnetic reconnec- 
tion. The derivation is analogous to the $3 \mathrm{D}$ viscous-resistive work by Westerberg and Akerstedt. ${ }^{2}$ For parts not covered in the present analysis, such as matching the solutions valid in the transition layer with analytical models for the magnetosheath flow, we refer to the original paper.

\section{A. The DeHoffmann-Teller velocity}

The absolute velocities in the main flow directions are divided into a DeHoffmann-Teller (HT) velocity ${ }^{14} U_{y, z}$ and the velocity with respect to the HT frame of reference $u_{y, z}$, such that

$$
\begin{aligned}
& \tilde{u}_{y}=u_{y}+U_{y}, \\
& \tilde{u}_{z}=u_{z}+U_{z} .
\end{aligned}
$$

The HT velocities are chosen such that

$$
\begin{aligned}
& E_{y}+B_{\xi}^{(0)} U_{z}=0, \\
& E_{z}-B_{\xi}^{(0)} U_{y}=0,
\end{aligned}
$$

where $B_{\xi}^{(0)}$ is to be defined in Sec. III B. This definition follows from $\nabla \times \mathbf{E}$, where

$$
\nabla \times \mathbf{E}=R^{1 / 2} \frac{\partial E_{z}}{\partial \xi} \hat{\mathbf{y}}-R^{1 / 2} \frac{\partial E_{y}}{\partial \xi} \hat{\mathbf{z}}=0 .
$$

This means that $E_{z}$ and $E_{y}$ are functions of only $y$ and $z$. As this is also valid for $B_{\xi}^{(0)}, 2$ it follows that $U_{y}$ and $U_{z}$ also depend only on $y$ and $z$.

\section{B. Governing equations and method of solution}

The MP transition layer separating the magnetosheath plasma from the magnetosphere plasma is treated as a resolution of a rotational discontinuity for which the plasma motion can be considered incompressible. The current transition layer is considered to correspond to the first large-amplitude Alfvén wave at the MP boundary. Heyn et al. ${ }^{15}$ showed that the Alfvén wave is followed by a series of compressible waves in order to match the lower plasma density in the magnetosphere; see Fig. 2. The plasma motion in accordance with this is considered to be governed by the incompressible MHD steady equation of motion (here in dimensionless form)

$$
(\mathbf{u} \cdot \nabla) \mathbf{u}=\frac{1}{R} \nabla^{2} \mathbf{u}-\nabla P+(\mathbf{B} \cdot \nabla) \mathbf{B},
$$

where $P$ is the total pressure,

$$
P=p+\frac{B^{2}}{2} \text {. }
$$

The magnetic field is described by the induction equation

$$
\frac{\partial \mathbf{B}}{\partial t}=\frac{1}{L_{u}} \nabla^{2} \mathbf{B}+\nabla \times(\mathbf{u} \times \mathbf{B})-c_{H} \nabla \times(\mathbf{j} \times \mathbf{B}) .
$$

To complete the system, we recall the divergence-free property of the velocity and magnetic field,

$$
\nabla \cdot \mathbf{u}=0
$$

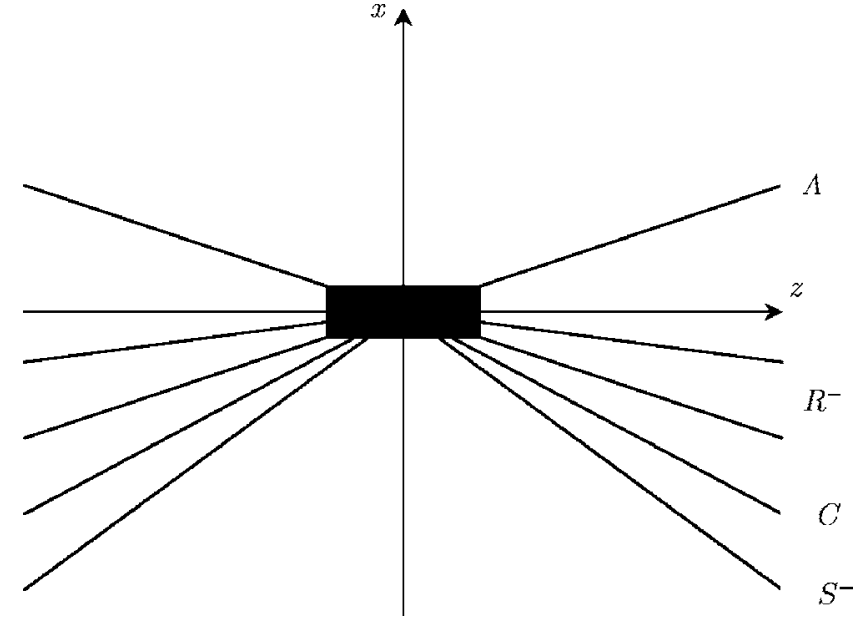

FIG. 2. MHD wave structure for steady-state magnetic reconnection at the dayside MP. The black box represents the diffusion region. This area is several orders smaller than the surrounding flow region. What happens inside the box is left out of the discussion. The main issue for the analysis is that reconnection does occur, not the onset physics in the diffusion area. The MP current layer is viewed as the first large-amplitude Alfvén wave at the MP boundary that rotates the magnetic field. In order to match the lower density in the magnetosphere, this wave is followed by a series of compressible waves. One possibility of the set of waves is shown in the figure where, going from the outside in, we first have the Alfvén wave $(A)$, a slow expansion fan $\left(R^{-}\right)$, a contact discontinuity $(C)$, and a slow shock $\left(S^{-}\right)$(Refs. 15 and 1).

$$
\nabla \cdot \mathbf{B}=0 .
$$

The dimensionless governing equations are solved approximately by applying the following perturbation expansion:

$$
\begin{aligned}
& u_{\xi}=R^{-1 / 4} \cdot u_{\xi}^{(0)}+R^{-1 / 2} \cdot u_{\xi}^{(1)}+\cdots, \\
& u_{y}=u_{y}^{(0)}+R^{-1 / 4} \cdot u_{y}^{(1)}+U_{y}+\cdots, \\
& u_{z}=u_{z}^{(0)}+R^{-1 / 4} \cdot u_{z}^{(1)}+U_{z}+\cdots, \\
& B_{\xi}=R^{-1 / 4} \cdot B_{\xi}^{(0)}+R^{-1 / 2} \cdot B_{\xi}^{(1)}+\cdots, \\
& B_{y}=B_{y}^{(0)}+R^{-1 / 4} \cdot B_{y}^{(1)}+\cdots, \\
& B_{z}=B_{z}^{(0)}+R^{-1 / 4} \cdot B_{z}^{(1)}+\cdots .
\end{aligned}
$$

The Lundqvist number is also a good choice of expansion parameter as it is large and can be considered to be of the same order of magnitude as the Reynolds number. The electric field and therefore the reconnection rate are considered to be of order $O\left(L_{u}^{-1 / 4}\right)$. It can, however, be shown that any other ordering that goes to zero slower than $O\left(L_{u}^{-1 / 2}\right)$ leads to the same set of equations. For instance, the reconnection rate by Petschek ${ }^{16}$ of order $O\left[\left(\log L_{u}\right)^{-1}\right]$ and the reconnection rate by Sonnerup ${ }^{17}$ of order $O(1)$ fulfill the requirement. Originally the magnetic Reynolds number $\left(R_{m}\right)$ is used to describe the Petschek reconnection rate. However, $R_{m}$ is normalized with a general characteristic velocity compared to $L_{u}$ where the corresponding velocity is the Alfvén velocity. As the reconnection rate for the present application at the dayside MP with asymmetric plasma densities in the magneto- 
sheath and the MP is not known to the authors, we will consider the ordering $O\left(L_{u}^{-1 / 4}\right)$.

\section{Equations governing the plasma flow north and south of the reconnection site}

The $y$ and $z$ component of the MHD equation of motion gives to order $O\left(R^{1 / 4}\right)$,

$$
\begin{aligned}
& u_{\xi}^{(0)} \frac{\partial u_{y}^{(0)}}{\partial \xi}=B_{\xi}^{(0)} \frac{\partial B_{y}^{(0)}}{\partial \xi}, \\
& u_{\xi}^{(0)} \frac{\partial u_{z}^{(0)}}{\partial \xi}=B_{\xi}^{(0)} \frac{\partial B_{z}^{(0)}}{\partial \xi} .
\end{aligned}
$$

Correspondingly, to order $O\left(R^{1 / 4}\right)$, the continuity equation (25) and the solenoidal property (26) of the magnetic field give

$$
\begin{aligned}
& u_{\xi}^{(0)}=u_{\xi}^{(0)}(y, z), \\
& B_{\xi}^{(0)}=B_{\xi}^{(0)}(y, z),
\end{aligned}
$$

that is, the lowest order of the tangential component of the velocity and the magnetic field is independent of $\xi$. To lowest order of the $y$ component of the induction equation (24), we get

$$
\begin{aligned}
& u_{y}^{(0)}=\frac{B_{y}^{(0)} \cdot u_{\xi}^{(0)}}{B_{\xi}^{(0)}}, \\
& u_{z}^{(0)}=\frac{B_{z}^{(0)} \cdot u_{\xi}^{(0)}}{B_{\xi}^{(0)}},
\end{aligned}
$$

which together with Eqs. (29), (30), and (28) gives two cases that we refer to as conditions north and south of the reconnection line, respectively

\section{Northern reconnection}

$$
\begin{gathered}
u_{\xi}^{(0)}=B_{\xi}^{(0)}, \\
u_{y}^{(0)}=B_{y}^{(0)}, \\
u_{z}^{(0)}=B_{z}^{(0)} .
\end{gathered}
$$

\section{Southern reconnection}

$$
\begin{aligned}
& u_{\xi}^{(0)}=-B_{\xi}^{(0)}, \\
& u_{y}^{(0)}=-B_{y}^{(0)}, \\
& u_{z}^{(0)}=-B_{z}^{(0)} .
\end{aligned}
$$

From the $\xi$ component of Eq. (22) to order $O\left(R^{0}\right)$ together with Eqs. (29) and (30) to order $O\left(R^{0}\right)$ we can verify one fundamental property of the transition layer

$$
\frac{\partial P}{\partial \xi}=0,
$$

i.e., the total pressure is constant through the transition layer. The solenoidal property of the magnetic field (26) and the continuity equation (25) to order $O\left(R^{0}\right)$ gives

$$
\begin{aligned}
& \frac{\partial B_{\xi}^{(1)}}{\partial \xi}+\frac{\partial B_{y}^{(0)}}{\partial y}+\frac{\partial B_{z}^{(0)}}{\partial z}=0, \\
& \frac{\partial u_{\xi}^{(1)}}{\partial \xi}+\frac{\partial u_{y}^{(0)}}{\partial y}+\frac{\partial U_{y}}{\partial y}+\frac{\partial u_{z}^{(0)}}{\partial z}+\frac{\partial U_{z}}{\partial z}=0 .
\end{aligned}
$$

In order to obtain the equations governing the plasma flow north of the reconnection site, we subtract Eq. (41) from Eq. (40) using the properties for the northern reconnection case (33)-(35). This results in the relation

$$
u_{\xi}^{(1)}-B_{\xi}^{(1)}=-\left(\frac{\partial U_{y}}{\partial y}+\frac{\partial U_{z}}{\partial z}\right) \cdot \xi+f(y, z)
$$

where the function $f$ can be chosen as zero. Differentiating the $y$ component of the induction equation (24) to order $O\left(R^{-1 / 2}\right)$ with respect to $\xi$, and subtracting the result from the $y$ component of the MHD equation of motion (22) to order $O\left(R^{0}\right)$, using Eqs. (33)-(35) and (42) results in the equation describing properties north of a reconnection line in the $y$ direction. The $z$ component is obtained through the same procedure as above, i.e., we subtract Eq. (41) from Eq. (40), using the properties for the northern reconnection case, Eqs. (33)-(35), resulting in Eq. (42). Differentiating the $z$ component of the induction equation (24) to order $O\left(R^{-1 / 2}\right)$ with respect to $\xi$, and subtracting the result from the $z$ component of the MHD equation of motion (22) to order $O\left(R^{0}\right)$, using Eqs. (33)-(35) we obtain the following system representing the $z$ and $y$ component, respectively:

$$
\begin{aligned}
& \hat{\mathbf{y}}: \quad \frac{\partial^{2}}{\partial \xi^{2}}\left(\alpha u_{y}^{(0)}+\underline{c_{H} B_{x}^{(0)} u_{z}^{(0)}}\right)+2 \xi\left(\frac{\partial U_{y}}{\partial y}+\frac{\partial U_{z}}{\partial z}\right) \frac{\partial u_{y}^{(0)}}{\partial \xi} \\
&+u_{y}^{(0)} \frac{\partial U_{z}}{\partial z}-2 U_{y} \frac{\partial u_{y}^{(0)}}{\partial y}-U_{y} \frac{\partial u_{z}^{(0)}}{\partial z}-U_{y} \frac{\partial U_{y}}{\partial y}-u_{z}^{(0)} \frac{\partial U_{y}}{\partial z} \\
&-U_{z}\left(\frac{\partial u_{y}^{(0)}}{\partial z}+\frac{\partial U_{y}}{\partial z}\right)-\frac{\partial P}{\partial y}=0, \\
& \hat{\mathbf{z}}: \quad \frac{\partial^{2}}{\partial \xi^{2}}\left(\alpha u_{z}^{(0)}-\frac{\left.c_{H} B_{x}^{(0)} u_{y}^{(0)}\right)}{\partial \xi}\left(\frac{\partial U_{y}}{\partial y}+\frac{\partial U_{z}}{\partial z}\right) \frac{\partial u_{z}^{(0)}}{\partial \xi}\right. \\
& \quad+u_{z}^{(0)} \frac{\partial U_{y}}{\partial y}-2 U_{z} \frac{\partial u_{z}^{(0)}}{\partial z}-U_{z} \frac{\partial u_{y}^{(0)}}{\partial y}-U_{y}\left(\frac{\partial u_{z}^{(0)}}{\partial y}+\frac{\partial U_{z}}{\partial y}\right) \\
& \quad-U_{z} \frac{\partial U_{z}}{\partial z}-u_{y}^{(0)} \frac{\partial U_{z}}{\partial y}-\frac{\partial P}{\partial z}=0 .
\end{aligned}
$$

Here $\alpha$ is chosen as 2, considering the Reynolds and Lundqvist number to be of the same order of magnitude. The underlined terms are as introduced in Sec. II, the additional terms originating from the inclusion of the Hall term in the generalized Ohm's law. These equations are identical to Eqs. (2.29) and (2.30) in Westerberg and Åkerstedt, ${ }^{2}$ with exception of the additional Hall terms [underlined in Eq. (43)]. This result can also be obtained using a solvability condition. ${ }^{1}$ We note that Eq. (43) is linear, in comparison to the nonlinear MHD equation of motion (22). 
Equation (43) yields both for the properties north and south of the reconnection site. Even if the equations seem identical for each case, the (general) HT velocity is different north and south of the reconnection site. ${ }^{2}$ The conditions north and south correspond to the following properties for the normal component of $\mathbf{B}$ :

North: $B_{x}^{(0)}>0$,

South: $B_{x}^{(0)}<0$.

Having obtained the equations governing the plasma flow through the MP north and south of the reconnection site, the next step is to find their solutions. This is presented in the following section.

\section{SOLUTIONS}

Once the governing equations for the plasma flow through the MP boundary during conditions of ongoing reconnection have been established, we continue with their solutions. As a first-order approximation we consider a constant HT velocity. In our previous work on the viscousresistive case, the HT were derived from outer ideal MHD models in which viscosity and resistivity were neglected. The same outer solutions can be applied for the present case, but since the only difference between the present case with the Hall term and the previous models is the structure of the transition region, we only consider the homogeneous solutions describing the structure of the transition. We note that Eq. (43) is in parabolic form and consider the velocity components to be in the form

$$
\begin{aligned}
& u_{z}^{(0)}=f(\eta), \\
& u_{y}^{(0)}=g(\eta),
\end{aligned}
$$

where

$$
\eta=\xi h(z)
$$

is the self-similar variable and $h(z)$ is the inverse of the transition layer thickness $\delta(z)$. Having $\mathbf{u}$ as a function of $\xi$ and $z$, $\partial / \partial y=0$. This reduces Eq. (43) to the system

$$
\begin{aligned}
& 2 U_{z} \frac{\partial u_{z}^{(0)}}{\partial z}=2 \frac{\partial^{2} u_{z}^{(0)}}{\partial \xi^{2}}-c_{H} B_{\xi}^{(0)} \frac{\partial^{2} u_{y}^{(0)}}{\partial \xi^{2}}, \\
& \left(U_{y}+U_{z}\right) \frac{\partial u_{y}^{(0)}}{\partial z}=2 \frac{\partial^{2} u_{y}^{(0)}}{\partial \xi^{2}}+c_{H} B_{\xi}^{(0)} \frac{\partial^{2} u_{z}^{(0)}}{\partial \xi^{2}} .
\end{aligned}
$$

Applying Eqs. (44)-(46) results in the following set of ODEs:

$$
\begin{aligned}
& f^{\prime \prime}+4 \eta f^{\prime}+\chi g^{\prime \prime}=0, \\
& g^{\prime \prime}+2 \eta g^{\prime}+a 2 \eta f^{\prime}-\chi f^{\prime \prime}=0,
\end{aligned}
$$

where

$$
a \equiv U_{y} / U_{z},
$$

$$
\chi \equiv \frac{1}{2} c_{H} B_{\xi}^{(0)} .
$$

$\chi$ is hence proportional to $c_{H}$. $B_{\xi}^{(0)}$ is a function of only $y$ and $z$, and can thus be considered to be constant through the layer. For the solution to Eq. (44), we consider

$$
\begin{aligned}
& f^{\prime}=A_{1} e^{-\alpha \eta^{2},}, \\
& g^{\prime}=A_{2} e^{-\alpha \eta^{2},},
\end{aligned}
$$

where $A_{1}, A_{2}$, and $\alpha$ are constants. Equation (49) in Eq. (47) results in

$$
\begin{aligned}
& A_{1}=\frac{\alpha \chi}{2-\alpha} A_{2}, \\
& A_{2}=-\frac{\alpha \chi+a}{1-\alpha} A_{1} .
\end{aligned}
$$

Solving for $\alpha$ gives

$$
\alpha^{2}+\frac{a \chi-3}{1+\chi^{2}} \alpha+\frac{2}{1+\chi^{2}}=0
$$

with the solution

$$
\begin{aligned}
& \alpha_{1}=\frac{3-a \chi}{2\left(1+\chi^{2}\right)}+\frac{1}{2\left(1+\chi^{2}\right)} \sqrt{1-6 a \chi+\left(a^{2}-8\right) \chi^{2}}, \\
& \alpha_{2}=\frac{3-a \chi}{2\left(1+\chi^{2}\right)}-\frac{1}{2\left(1+\chi^{2}\right)} \sqrt{1-6 a \chi+\left(a^{2}-8\right) \chi^{2}} .
\end{aligned}
$$

Equation (49) can then be expanded such that

$$
\begin{aligned}
& f^{\prime}=A_{1} e^{-\alpha_{1} \eta^{2}}+B_{1} e^{-\alpha_{2} \eta^{2}}, \\
& g^{\prime}=A_{2} e^{-\alpha_{1} \eta^{2}}+B_{2} e^{-\alpha_{2} \eta^{2}},
\end{aligned}
$$

where $A_{1}, A_{2}, B_{1}$, and $B_{2}$ are constants. This gives in turn that

$$
\begin{aligned}
u_{z}^{(0)}=f(\eta)= & A_{1} \sqrt{\frac{\pi}{2 \alpha_{1}}} \operatorname{erf}\left(\sqrt{\alpha_{1}} \eta\right) \\
& +B_{1} \sqrt{\frac{\pi}{2 \alpha_{2}}} \operatorname{erf}\left(\sqrt{\alpha_{2}} \eta\right)+C_{1}, \\
u_{y}^{(0)}=g(\eta)= & A_{2} \sqrt{\frac{\pi}{2 \alpha_{1}}} \operatorname{erf}\left(\sqrt{\alpha_{1}} \eta\right) \\
& +B_{2} \sqrt{\frac{\pi}{2 \alpha_{2}}} \operatorname{erf}\left(\sqrt{\alpha_{2}} \eta\right)+C_{2} .
\end{aligned}
$$

We consider the following matching conditions:

$$
\begin{aligned}
\eta \rightarrow+\infty: & u_{z}^{(0)} \rightarrow u_{z}^{\text {outer }}, \\
& u_{y}^{(0)} \rightarrow u_{y}^{\text {outer }}, \\
\eta \rightarrow-\infty: & u_{z}^{(0)} \rightarrow u_{z}^{\text {inner }}, \\
& u_{y}^{(0)} \rightarrow u_{y}^{\text {inner }},
\end{aligned}
$$

where outer and inner correspond to the values of the velocity components at respective sides of the magnetopause 
boundary, i.e., in the magnetosheath and in the magnetosphere, respectively. In order to compare the analytical results with in situ measurements, these values are brought from spacecraft data during a magnetopause crossing. See Sec. V B for further details of the observational coupling. Equation (54) together with Eq. (55) then gives

$$
\begin{aligned}
u_{z}^{(0)}=f(\eta)= & \frac{\frac{1}{2} \Gamma+K_{2}}{K_{2}-K_{1}} \operatorname{erf}\left(\sqrt{\alpha_{1}} \eta\right)+\frac{\frac{1}{2} \Gamma+K_{1}}{K_{2}-K_{1}} \operatorname{erf}\left(\sqrt{\alpha_{2}} \eta\right) \\
u_{y}^{(0)}=g(\eta)= & -\frac{K_{1}\left(\frac{1}{2} \Gamma+K_{2}\right)}{K_{2}-K_{1}} \operatorname{erf}\left(\sqrt{\alpha_{1}} \eta\right) \\
& +\frac{K_{2}\left(\frac{1}{2} \Gamma+K_{1}\right)}{K_{2}-K_{1}} \operatorname{erf}\left(\sqrt{\alpha_{2}} \eta\right)+\frac{\Gamma}{2}
\end{aligned}
$$

Here

$$
\begin{array}{r}
K_{1}=\frac{a+\frac{\alpha_{1} \chi}{1+\chi^{2}}}{\frac{\alpha_{1}}{\left(1+\chi^{2}\right)}-1}, \\
K_{2}=\frac{a+\frac{\alpha_{2} \chi}{1+\chi^{2}}}{\frac{\alpha_{2}}{\left(1+\chi^{2}\right)}-1},
\end{array}
$$

where $\alpha_{1}$ and $\alpha_{2}$ are in accordance with Eq. (52). With $K_{1}$ and $K_{2}$ we now have complete lower-order approximations for the velocity and magnetic field development through the MP transition layer. $\mathbf{u}$ and $\mathbf{B}$ are functions of the parameters $a$ and $\chi$ defined in Eq. (48).

Recalling that the (anomalous) viscosity is included in the transverse variable $\eta$, we can introduce a parameter $\lambda$ that is a scale factor to a characteristic viscosity value, such that

$$
u_{z}^{(0)}=f(\eta)=\frac{\frac{1}{2} \Gamma+K_{2}}{K_{2}-K_{1}} \operatorname{erf}\left(\sqrt{\alpha_{1}} \lambda \eta\right)+\frac{\frac{1}{2} \Gamma+K_{1}}{K_{2}-K_{1}} \operatorname{erf}\left(\sqrt{\alpha_{2}} \lambda \eta\right)
$$

$$
\begin{aligned}
u_{y}^{(0)}=g(\eta)= & -\frac{K_{1}\left(\frac{1}{2} \Gamma+K_{2}\right)}{K_{2}-K_{1}} \operatorname{erf}\left(\sqrt{\alpha_{1}} \lambda \eta\right) \\
& +\frac{K_{2}\left(\frac{1}{2} \Gamma+K_{1}\right)}{K_{2}-K_{1}} \operatorname{erf}\left(\sqrt{\alpha_{2}} \lambda \eta\right)+\frac{\Gamma}{2} .
\end{aligned}
$$

$\lambda$ then serves as an optimization parameter in order to fit Eq. (58) to observations. See Sec. V B.

\section{RESULTS}

Having obtained the solution for the velocity and magnetic field development through the MP boundary, we continue by plotting the solutions for typical parameter values. The parameter $\chi\left(\equiv \frac{1}{2} c_{H} B_{\xi}^{(0)}\right)$ is proportional to the relation between the Hall and viscous terms. Having a plasma that is mainly collisionless, the nonideal effects of viscosity and resistivity are viewed as anomalous transport parameters. These develop through wave/particle interactions in the plasma, and have values that are highly uncertain. This gives us space to vary the value of the parameter $\chi$ rather widely. With this in mind, we consider the following parameter values in addition to the matching conditions (55):

$$
\begin{aligned}
& V_{A}=250 \mathrm{~km} / \mathrm{s}, \\
& n=10 \mathrm{~cm}^{-3}, \\
& a=0.5,
\end{aligned}
$$

where $a$ is according to Eq. (48a).

\section{A. Solution regimes for different values of $\chi$}

For $\chi \rightarrow 0, \chi=0.3 ; 1 ; 5$, and the parameter values listed in Eq. (59), we obtain the development of the plasma velocity during the transition of the MP transition layer under conditions of ongoing magnetic reconnection. The results are presented in Fig. 3 together with in situ measurements (see Sec. V B). In Fig. $4, u_{z}^{(0)}$ is plotted versus $u_{y}^{(0)}$ for the corresponding set of values for $\chi$. Having a constant HT velocity, the result is independent of the location north or south of the reconnection site. While $\mathbf{u}^{(0)}= \pm \mathbf{B}^{(0)}$ (positive sign to the north), ${ }^{2}$ the structural behavior is the same both for the velocity in the HT frame and the magnetic field. In this section, we therefore present the results for the velocity $\mathbf{u}$.

The limit $\chi \rightarrow 0$ corresponds to the pure viscous-resistive solutions. It is evident that as the Hall term $c_{H}$ grows, the flow behavior becomes more perturbed. Figure 3 clearly shows the increasing influence of the Hall physics on the global flow behavior as the plasma moves through the MP. The effect of the growing influence of the Hall current is also viewed in the hodograph plots in Fig. 4.

\section{B. Comparison with in situ measurements}

The data for the observational comparison come from an MP crossing made by the Cluster spacecraft armada on January 26, 2001. During that day, the Cluster fleet was skimming the MP surface for a period of several hours. These MP crossings have also been investigated by Phan et al. ${ }^{18}$ in their study of continuous reconnection. Westerberg et al. ${ }^{19}$ have also used data from these events when performing a coupling between in situ measurements and the 3D viscous-resistive reconnection model of which the present analysis is a further development. The present comparison with Cluster data is considered to be a first simple case study approach, in order to illustrate the correlation between the analytical model and in situ measurements. Since the present approach has its focus on the Hall physical effects in connection with magnetic reconnection, i.e., not primarily being an observational study of MP crossings, we refer to the work by Phan et al. ${ }^{18}$ for excessive proof of ongoing magnetic reconnection on the actual date. Also, for further information about the Cluster mission such as the onboard instrumentation and spacecraft orbit, we refer to the work of, e.g., Escoubet et al. ${ }^{20,21}$ In a development of the present paper, it is of course of great 

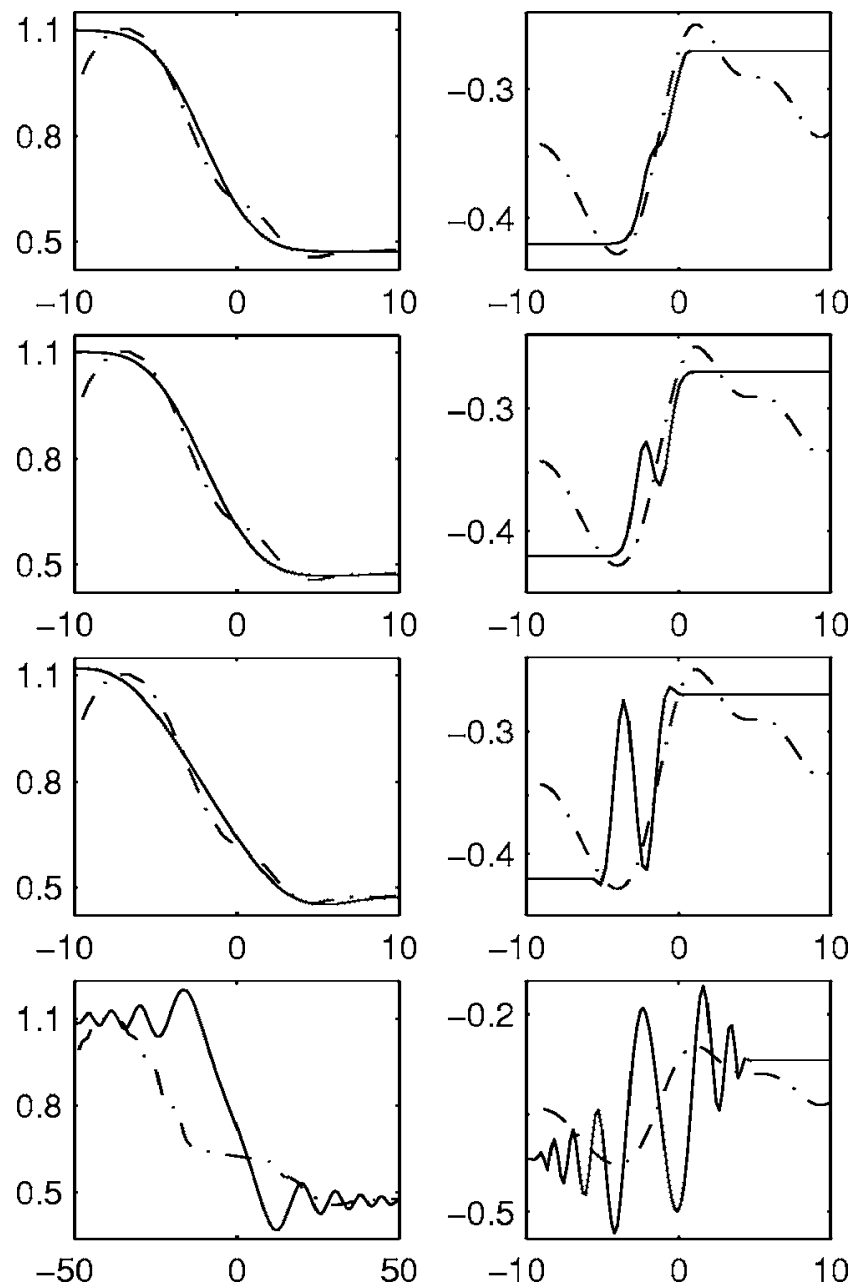

FIG. 3. Development of the plasma velocity (dimensionless quantity) through the transition of the MP as a function of the self-similar variable $\eta$. Solid line: analytical solution. Dash-dotted line: observation by Cluster spacecraft 4, made on 26 January 2001, 11:02:45-11:04:21 Universal Time (UT). Left column: $u_{z}^{(0)}$. Right column: $u_{y}^{(0)}$. Respective rows correspond to $\chi=0 ; 0.3 ; 1 ; 5$, respectively, where the top row represents $\chi=0$ and the bottom row $\chi=5$.

interest to extend the observational part to more MP crossings in order to obtain a more thorough statistical base.

Figure 3 shows the observed velocity and the analytical results. The Cluster data are transformed to the local MP plane coordinates using the method of maximum variance analysis on the electric field (MVAE), which for the actual MP crossing gives a well determined boundary normal. We see that the analytical solution for the $z$ component satisfies the corresponding observation rather well as $\chi \leqslant 1$. As $\chi$ have reached five and thus the Hall term is dominating over the viscous-resistive term, large-scale oscillations have been brought into the system. The transition region has also increased significantly compared to the case $\chi=1$. For the $y$ component, the Hall term affects the solution at an earlier stage compared to the $z$ component. Here the oscillating behavior is connected with the transition region rather than the regions on respective sides of the current sheet.

The comparison in the present case is not to be considered as a quantitative quality check of the analytical modelthat is left for a development of the present analysis in which
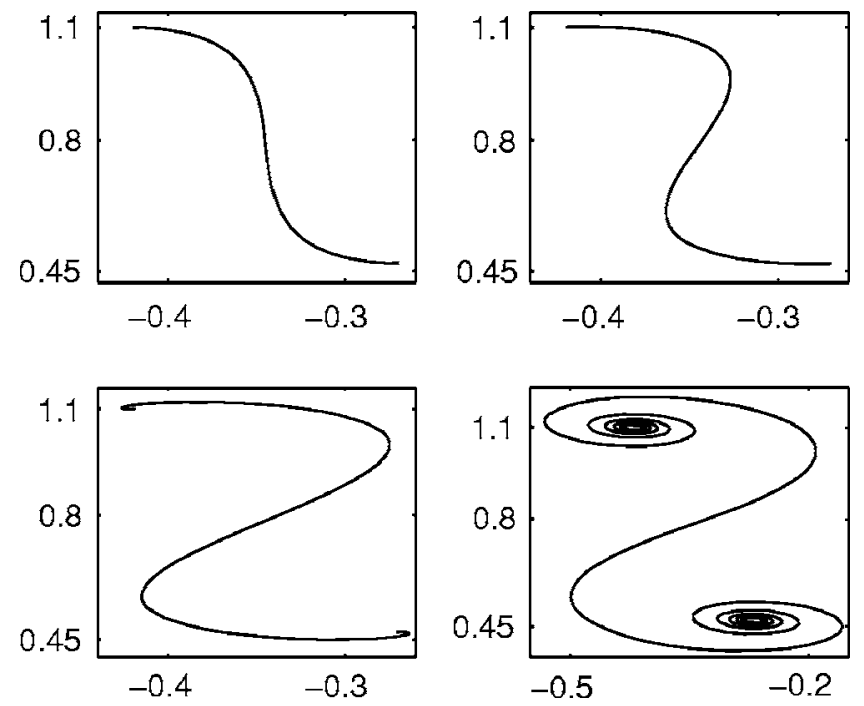

FIG. 4. Hodograph plot, where $u_{z}^{(0)}$ is plotted versus $u_{y}^{(0)}$ for (from upper left to lower right) $\chi=0 ; 0.3 ; 1 ; 5$. Upper left represents the viscous-resistive solution corresponding to $\chi=0$. Lower right corresponds to $\chi=5$.

more MP crossings will be treated. Nevertheless, these results indicate that the analytical model does manage to resolve the main structural development during the transition of the MP boundary. In order to develop the present study in terms of optimizing the fit between observed data and analytical solutions, it is natural to consider the same methodology as that utilized by Westerberg et al. ${ }^{19}$ where the whole analytical solution is fitted to spacecraft data in a leastsquares sense. This is particularly good when estimating the actual value of the resistive parameters of anomalous viscosity and resistivity. For the present study where we use a simplified coupling approach to see to what extent our model follows the main characteristics of in situ measurements, the present methodology is sufficient as a first-order approximation.

\section{Discussion}

Having obtained the solution and plotted the results for the plasma flow, we clearly see from Figs. 3 and 4 that the structural behavior significantly changes as the value of $\chi$ increases. The influence of the Hall physics on the reconnection dynamics has been shown in several studies to be dependent on the relation between the Hall parameter $c_{H}$ and the inverse of the Lundqvist number $L_{u}{ }^{5,8,22,6}$ Especially when $c_{H}^{2}>L_{u}^{-1}$, the Hall effect has been shown to dominate significantly. It is also shown that the inclusion of the Hall term brings the dynamics of Whistler waves into the system. Craig et al. ${ }^{5}$ explicitly demonstrated that for a dominating Hall parameter, large-scale oscillations are established in the fields. The Hall currents have been shown to play a significant role in the magnetic merging solutions both for reconnection at the Solar corona and for applications at the terrestrial MP. ${ }^{5,22}$ The references within this section clearly show that the small-scale reconnection dynamics changes dramatically as the Hall term $\left(c_{H}\right)$ grows. We see from Figs. 3 and 4 that as the Hall effect is much smaller than the viscous/ resistive [recalling $O(R)=O\left(L_{u}\right)$ ] effect, the solutions show 
the same behavior as the viscous-resistive solutions, as should be the case. As $c_{H}$ approaches the order of the Reynolds number, the Hall effect begins to significantly influence the flow pattern. When $\chi$ increases to 5, the $y$ component of the plasma velocity loses all of its initial structure, while the situation is not as dramatic for the $z$ component. In Fig. 3, steady oscillations are seen in the $z$ component. The effect is similar for the $y$ component, but here the oscillations propagate throughout the layer. In addition to the dramatic structural change for increasing $c_{H}$, the MP transition layer thickness is also affected as $c_{H}$ begins to dominate over the viscous-resistive effects. This is viewed in Fig. 3 as a thickening of the transition region-an effect best illustrated by $u_{z}^{(0)}$, since $u_{y}^{(0)}$ undergoes such a significant change with increasing oscillations both in the outer fields and through the main current sheet.

As discussed in the Introduction, it has been suggested by several authors that the Hall effects become dominant when $c_{H}>L_{u}^{-1}$, with an even more distinctive change in the reconnection topology and the surrounding plasma structure when $c_{H}^{2}>L_{u}^{-1}$. Craig et al. ${ }^{5}$ conclude that the latter relation is of greater significance than the first. Their model is, however, based on a collision-dominated reconnection process in the Solar corona. Nevertheless, it is of interest to couple its relevance to our application of reconnection at the dayside MP boundary. From the present analysis, we conclude that the Hall parameter scales inversely to the Lundqvist number, i.e., $c_{H} \approx 1 / L_{u}$. The change in flow structure presented in Figs. 3 and 4 shows that when $c_{H}$ approaches $L_{u}(\chi=1)$, the flow behavior starts to break from the initial structure corresponding to the viscous-resistive solution. The first signature of the Hall effect onto the velocity development through the transition layer occurs when $\chi \approx 0.3$. It is noticeable that the effect is stronger as $\chi$ grows from 5 to 10 than for the corresponding increase from 1 to 5 . The critical relation between the Hall parameter and the Lundqvist number, $c_{H}$ $>L_{u}^{-1}$, can thus be concluded to be a condition that is valid for the influence of the Hall current onto the plasma flow dynamics near a reconnection site.

In addition to analytical approaches of the Hall influence onto the reconnection process, there have been many simulations made during the past decade [see, e.g., in addition to the work presented in Sec. I and earlier in this section, Hall MHD (HMHD) simulations made by $\mathrm{Ma}$ and Bhattacharjee, ${ }^{23}$ Lottermoser and Scholer, ${ }^{24}$ and Morales et $a{ }^{25}{ }^{25}$. Comparing the obtained result with the work by Morales et al., ${ }^{25}$ who applied HMHD simulations onto the MP current sheet, shows that the structural behavior is analogous in terms of flux pileup for increasing value of the Hall parameter. The oscillating behavior shown in the present analysis, which is very significant for $\chi>5$, is, however, not caught in the simulations. This is due to the dominating value of $\chi\left(=\frac{1}{2} c_{H} B_{\xi}^{(0)}\right)$. Morales et al., ${ }^{25}$ however, use a maximum value corresponding to $c_{H}=1$ while for the present approach we investigate the Hall influence for up to $\chi \approx 5$, which corresponds to a value of $c_{H}$ that is significantly higher than 1. The oscillating behavior for a dominating Hall parameter as discussed earlier in this section has also been observed by Craig et al. ${ }^{5}$ One explanation for the occurrence of oscillations can also be the combination of resistive and Hall physical effects.

Comparing the analytical results with spacecraft observations (cf. Fig. 3), we see that for the actual MP crossing, the plasma velocity varies without any significant oscillating behavior. Whether this structural development is dominating is to be left for a more statistical observational/analytical approach. Possible error sources for the mismatch between the analytical and observed values can be local effects, causing the 1D stationary current sheet assumption upon which the observational analysis is based to not be entirely valid for the present MP crossing. Phan et al. ${ }^{18}$ also suggest that plasma blobs occur along the MP surface, which of course significantly contribute to a perturbation of the initial assumption of 1D stationarity. In addition, other local effects that not are resolved by the analytical model also affect the outcome of the comparison.

Finally, it may be of interest to see what the condition $c_{H}>L_{u}^{-1}$ means in terms of the anomalous magnetic diffusivity and viscosity. Considering Eq. (15), we obtain from $c_{H}$ $>L_{u}^{-1}$ the condition [using the parameter values defined in Eq. (59)]

$$
\eta_{d}<\frac{B_{0}}{n e \mu_{0}} \approx 5 \times 10^{3} \mathrm{~km}^{2} / \mathrm{s},
$$

which is within the range of earlier reported estimates by Axford, ${ }^{26}$ Haerendel et al. ${ }^{27}$ and Westerberg et al. ${ }^{19}$

\section{SUMMARY AND CONCLUSIONS}

In this three-dimensional analysis, we have investigated how the inclusion of the Hall term in the generalized Ohm's law affects the plasma flow in the vicinity of a reconnection site at the dayside MP. The analytical model is compared with in situ measurements made by the Cluster armada during one MP crossing. The governing equations are solved approximately with the use of an ordinary perturbation technique with expansions in order of large Reynolds and Lundqvist numbers together with a small parameter expansion in $c_{H}$. It has been shown in several recent studies that the Hallphysical effects are dominating when the ion-skin depth is greater than the inverse of the Lundqvist number. Here we show that the solutions for a very small value of the Hall parameter are identical to the viscous-resistive solutions. The effect of the Hall physics on the plasma flow becomes visible as the Hall term approaches the order of the Lundqvist number. Furthermore, it is shown that the overall large-scale dynamics is strongly dependent on the magnitude of the Hall parameter. Starting with a pure viscous-resistive solution, we investigate the influence of the Hall parameter as the value is increased. This introduces perturbations onto the initial flow structure in the form of oscillations in the fields, and a thickening of the MP transition layer. It is shown that Hall effects have been established in the flow when the Hall parameter is of the same order as the inverse of the Lundqvist number. 
For larger values of the Hall parameter, the initial flow structure experiences a major change.

The inclusion of the Hall physics has been shown in several studies to have a major impact on the small-scale reconnection dynamics in the diffusion region. Here we have put the context out of the diffusion region and presented solutions that show that the Hall term can also affect the structure of the rotational discontinuity far outside the diffusion region.

There are several ways to go in order to extend the present analysis: as for the viscous-resistive 3D model, it is highly interesting to couple the results with global MHD simulations and satellite observations. Furthermore, in order to put the analytical results into a more statistical relation with spacecraft observations, it is a straightforward continuation to let the coupling analysis comprise several more MP crossings.

\section{ACKNOWLEDGMENTS}

This research project is financed through the Swedish National Graduate School of Space Technology.

${ }^{1}$ L. G. Westerberg and H. O. Åkerstedt, J. Plasma Phys. 71, 537 (2005).

${ }^{2}$ L. G. Westerberg and H. O. Åkerstedt, J. Plasma Phys. 72, 359 (2006).

${ }^{3}$ L. G. Westerberg and H. O. Åkerstedt, J. Plasma Phys. 73, 145 (2007).

${ }^{4}$ L. G. Westerberg and H. O. Åkerstedt, J. Plasma Phys. 73, 89 (2007).

${ }^{5}$ I. J. D. Craig, J. Heerikhuisen, and P. G. Watson, Phys. Plasmas 10, 3120 (2003).

${ }^{6}$ T. Senanayake and I. J. D. Craig, Astron. Astrophys. 451, 1117 (2006).

${ }^{7}$ J. A. Breslau and S. C. Jardin, Phys. Plasmas 10, 1291 (2003).

${ }^{8}$ J. D. Huba and L. I. Rudakov, Phys. Rev. Lett. 93, 175003 (2004).

${ }^{9}$ A. Vaivads, Y. Khotyaintsev, M. André, A. Retinó, S. C. Buchert, B. N.
Rogers, P. Décréau, G. Paschmann, and T. D. Phan, Phys. Rev. Lett. 93, 105001 (2004).

${ }^{10}$ L. G. Westerberg, J. Vedin, A. Ekenbäck, and H. O. Åkerstedt, "Threedimensional flow near a reconnection site at the dayside magnetopause: analytical solutions coupled with MHD simulations," J. Plasma Phys. (in press).

${ }^{11}$ D. A. Gurnett and A. Bhattacharjee, Introduction to Plasma Physics (Cambridge University Press, Cambridge, UK, 2005), p. 244.

${ }^{12}$ T. J. M. Boyd and J. J. Sanderson, Plasma Dynamics (Nelson, London, UK, 1969), p. 48.

${ }^{13}$ C. T. Russell and R. C. Elphic, Space Sci. Rev. 22, 681 (1978).

${ }^{14}$ F. DeHoffmann and E. Teller, Phys. Rev. 80, 692 (1950).

${ }^{15}$ M. F. Heyn, H. K. Biernat, V. S. Semenov, and I. V. Kubyshkin, J. Geophys. Res. 90, 1781 (1985).

${ }^{16}$ H. Petschek, NASA Spec. Publ. SP-50 (1964), pp. 425-439.

${ }^{17}$ B. U. Sonnerup, J. Plasma Phys. 4, 161 (1970).

${ }^{18}$ T. D. Phan, M. W. Dunlop, G. Paschmann, B. Klecker, J. M. Bosqued, H. R. Rème, A. Balogh, C. Twitty, F. S. Mozer, C. W. Carlson, C. Mouikis, and L. M. Kistler, Ann. Geophys. 22, 2355 (2004).

${ }^{19}$ L. G. Westerberg, H. O. Åkerstedt, H. Nilsson, H. Rème, and A. Balogh, J. Geophys. Res. (submitted).

${ }^{20}$ C. P. Escoubet, R. Schmidt, and M. L. Goldstein, Space Sci. Rev. 79, 11 (1997).

${ }^{21}$ C. P. Escoubet, M. Fehringer, and M. Goldstein, Ann. Geophys. 19, 1197 (2001).

${ }^{22}$ J. Birn, J. F. Drake, M. A. Shay, B. N. Rogers, R. E. Denton, M. Hesse, M. Kuznetsova, Z. W. Ma, A. Bhattacharjee, A. Otto, and P. L. Pritchett, J. Geophys. Res. 106, 3715, DOI:10.1029/1999JA900449 (2001).

${ }^{23}$ Z. W. Ma and A. Bhattacharjee, J. Geophys. Res. 106, 3773, DOI:10.1029/1999JA001004 (2001).

${ }^{24}$ R.-F. Lottermoser and M. Scholer, J. Geophys. Res. 102, 4875, DOI:10.1029/96JA03634 (1997).

${ }^{25}$ L. F. Morales, S. Dasso, and D. O. Gómez, J. Geophys. Res. 110, A04204, DOI:10.1029/2004JA010675 (2005).

${ }^{26}$ W. I. Axford, Planet. Space Sci. 12, 45 (1964).

${ }^{27}$ G. Haerendel, N. Paschmann, N. Sckoke, and H. Rosenbauer, J. Geophys. Res. 82, 3195 (1978). 